\title{
ON INTEGRAL REPRESENTATIONS
}

\author{
BY ANDREAS DRESS
}

Communicated by Hyman Bass, April 1, 1969

Let $G$ be a finite group and $p$ a prime. $G$ is called cyclic $\bmod p$ if there exists a normal $p$-subgroup $N \unlhd G$ such that $G / N$ is cyclic.

Let $R$ be a commutative ring with $1 \in R$. Write $\mathfrak{C}_{R}(G)$ for the set of subgroups $U \leqq G$, which are cyclic $\bmod p$ for some appropriate prime $p(=p(U))$ with $p R \neq R$.

An $R G$-module $M$ is a finitely generated $R$-module, on which $G$ acts from the left by $R$-automorphisms. If $U \leqq G$ we write $\left.M\right|_{U}$ for the $R U$-module, one gets by restricting the action of $G$ on the $R$ module $M$ to $U$.

If $N$ is an $R U$-module, we write $N^{U \rightarrow G}$ for the induced $R G$-module $R G \otimes_{R U} N$.

Two $R G$-modules $M, N$ are called weakly isomorphic, if there exists an $R G$-module $L$ and a natural number $k$, such that $k \cdot M \oplus L$ $\cong k \cdot N \oplus L$ ( $k \cdot M$ short for $M \oplus \cdots \oplus M, k$ times), we write then $M \simeq N$.

REMARK. If the Krull-Schmidt-Theorem holds for $R G$-modules, we have

$$
M \simeq N \Leftrightarrow M \cong N .
$$

Theorem 1. Let $M, N$ be two RG-modules. If $\left.\left.M\right|_{U} \simeq N\right|_{v}$ for all $U \in \mathfrak{C}_{R}(G)$, then $M \simeq N$. Moreover there exist for any $U \in \mathfrak{C}_{R}(G)$ two $R$-free $R G$-modules $M(U), N(U)$ with $\left.\left.M(U)\right|_{V} \cong N(U)\right|_{V}$ for all $V \leqq G$, which do not contain any conjugate of $U$, but $\left.\left.M(U)\right|_{U} \not N(U)\right|_{U}$.

One can get an even more precise statement by using Grothendieckrings: Let $X(G, R)$ be the Grothendieck-ring of $R G$-modules with respect to split-exact sequences, i.e. $X(G, R)$ is an as additive group isomorphic to the free abelian group, generated by the isomorphism classes of $R G$-modules modulo the subgroup generated by all expressions of the form $M-M_{1}-M_{2}$ with $M \cong M_{1} \oplus M_{2}$-and the multiplication in $X(G, R)$ is given by the tensor-product $\otimes_{R}$ of $R G$-modules. Write $X_{Q}(G, R)$ for $Q \otimes X(G, R)$. Obviously $M \simeq N$ if and only if $M$ and $N$ represent the same element in $X_{Q}(G, R)$.

$X(\cdot, R)$ and $X_{Q}(\cdot, R)$ are obviously contravariant functors from the category of groups into the category of commutative rings. Especially for $U \leqq G$ one has restriction homomorphisms res $\left.\right|_{U}: X(G, R)$ $\rightarrow X(U, R), X_{Q}(G, R) \rightarrow X_{Q}(U, R)$ and Theorem 1 reads now 
Theorem $1^{\prime} .\left.\prod_{U \in \mathfrak{C}_{R}(G)} \operatorname{res}\right|_{U}: X_{Q}(G, R) \rightarrow \prod_{U \in \mathfrak{C}_{R}(G)} X_{Q}(U, R)$ is injective.

One can also describe the image of $X_{Q}(G, R)$ in $\prod_{U \in \mathfrak{K}_{R}(G)} X_{Q}(U, R)$. More generally let $\mathfrak{u}$ be any family of subgroups of $G$ closed with respect to subconjugation, i.e.

$$
U, V \leqq G, \quad g \in G, \quad g V g^{-1} \leqq U \in \mathfrak{U}
$$

implies $V \in \mathfrak{U}$. For any such triple $U, V \in \mathfrak{U}$ and $g \in G$ with $g V g^{-1} \leqq U$ one has a diagram:

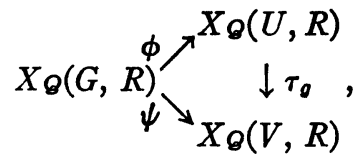

the maps $\phi$ and $\psi$ given by restriction, the map $\tau_{0}$ defined by $V \rightarrow U$, $v \rightarrow g v g^{-1}$, and one can easily see, that this diagram is commutative. Thus $\left.\prod_{v \in \mathfrak{u} \text { res }}\right|_{v}: X_{Q}(G, R) \rightarrow \prod_{U \in \mathfrak{u} X_{Q}}(U, R)$ maps $X_{Q}(G, R)$ into$$
X_{Q}(\mathfrak{U}, R)=\left\{\left(x_{U}\right)_{U \in \mathfrak{U}} \in \prod_{U \in \mathfrak{u}} X_{Q}(U, R) \mid \tau_{\theta}\left(x_{U}\right)=x_{V},\right.
$$$$
\text { whenever } \left.U, V \in \mathfrak{u}, g \in G \text { and } g V g^{-1} \leqq U\right\}
$$

and one has actually

Theorem 2. The canonical map $X_{Q}(G, R) \rightarrow X_{Q}(\mathfrak{U}, R)$ is always epimorphic and is an isomorphism if and only if $\mathfrak{u} \geqq \mathfrak{夭}_{R}(G)$.

It seems to be difficult, to prove a similar statement for $X(G, R)$ instead of $X_{Q}(G, R)$, but if $X^{\prime}(G, R)$ denotes the image of $X(G, R)$ in $X_{Q}(G, R)$, i.e. $X(G, R)$ mod torsion, and if for any subconjugately closed family $\mathfrak{U}$ of subgroups of $G$ we write $\mathfrak{V} \mathfrak{U}$ for $\{V \leqq G \mid$ there exists $U \unlhd V, U \in \mathfrak{U}, V / U$ a $p$-group $\}$ then one can prove

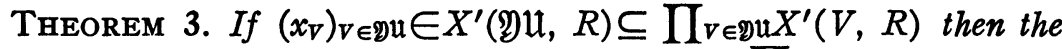
projection $\left(x_{U}\right)_{U \in \mathfrak{U}}$ of $\left(x_{V}\right)_{V \in \mathfrak{D} \mathfrak{u}}$ into $X^{\prime}(\mathfrak{U}, R) \subseteq \prod_{U \in \mathfrak{u}} X^{\prime}(U, R)$ is contained in the image of $X^{\prime}(G, R)$ in $X^{\prime}(\mathfrak{U}, R)$.

REMARK. One can form a category $\mathfrak{H}$, whose objects are the subgroups in $\mathfrak{U}$ with $\operatorname{Hom}_{\mathfrak{u}}(V, U)=\left\{g \in G \mid g V g^{-1} \leqq U\right\}$ and obvious composition. Then $\left.X(\cdot, R), X_{Q}(\cdot, R), X^{\prime}, R\right)$ are contravariant functors on $\mathfrak{U}$ and one has 


$$
\begin{gathered}
X(\mathfrak{U}, R)=\operatorname{proj} \lim X(\cdot, R), \quad X_{\boldsymbol{Q}}(\mathfrak{U}, R)=\underset{\mathfrak{u}}{\operatorname{proj}} \lim X_{\boldsymbol{Q}}(\cdot, R), \\
X^{\prime}(\mathfrak{U}, R)=\underset{\mathfrak{u}}{\operatorname{proj}} \lim X^{\prime}(\cdot, R) .
\end{gathered}
$$

We will state one lemma, which is fundamental for the proof of the above theorems.

We say, that an $R G$-module $M$ is weakly, resp. quasi- $\mathfrak{U}$-induced, if there exists a natural number $k$ and for any $U \in \mathfrak{U}$ two $R U$-modules $N_{1}(U), N_{2}(U)$ such that

$$
\begin{gathered}
k \cdot M \oplus \underset{U \in \mathfrak{U}}{\bigoplus} N_{1}(U)^{U \rightarrow G} \cong \underset{U \in \mathfrak{U}}{\bigoplus} N_{2}(U)^{U \rightarrow G}, \\
\text { resp. } k \cdot\left(M \oplus \underset{U \in \mathfrak{U}}{\bigoplus} N_{1}(U)^{U \rightarrow G}\right) \cong k \cdot\left(\bigoplus_{U \in \mathfrak{U}} N_{2}(U)^{U \rightarrow G}\right) .
\end{gathered}
$$

For a $G$-set $S$ (i.e. a finite set, on which $G$ acts from the left by permutations) we write $R[S]$ for the free $R$-module, generated by $S$, considered as $R G$-module by extending the action of $G$ on the basis $S$ linearly to $R[S]$. Two $G$-sets $S_{1}, S_{2}$ are $\mathfrak{U}$-isomorphic $\left(S_{1} \stackrel{\mathfrak{u}}{=} S_{2}\right)$, if the restrictions $S_{1} \mid v$ and $\left.S_{2}\right|_{U}$ to any $U \in \mathfrak{U}$ are isomorphic. Then we have the following

LEMMA. For a group $G$, a family $\mathfrak{U}$ of subgroups and a commutative ring $R$ the following four statements are equivalent:

(i) the trivial $R G$-module $R$ is weakly $\mathfrak{U}$-induced;

(ii) any $R G$-module is weakly $\mathfrak{U}$-induced;

(iii) $X_{Q}(G, R) \rightarrow \prod_{U \in \mathfrak{u}} X_{Q}(U, R)$ is injective;

(iv) if $S_{1}, S_{2}$ are two $\mathfrak{U}$-isomorphic $G$-sets, then $R\left[S_{1}\right] \simeq R\left[S_{2}\right]$.

Any of these statements implies, that every $R G$-module is quasi- $\mathfrak{Y} \overline{\mathfrak{u}}-$ induced with $\overline{\mathfrak{u}}=\left\{V \leqq G \mid\right.$ there exists $g \in G, U \in \mathfrak{U}$ with $\left.g V g^{-1} \leqq U\right\}$, i.e. the subconjugate closure of $\mathfrak{U}$ and $\mathfrak{V} \overline{\mathfrak{U}}=\{W \leqq G \mid$ there exists $V \in \overline{\mathfrak{u}}, V \unlhd W, W / V$ a $p$-group $\}$ (just as before). Especially any $R G$ module is quasi- $\mathfrak{V} \mathfrak{S}_{R}(G)$-induced-which generalizes a well-known result of Brauer-Bermann-Witt-Swan for the case $R=Q$. In case $\zeta$ is a $e$ th root of unity with $e=\exp (G)$ and there is a homomorphism $Z[\zeta] \rightarrow R$ one can sharpen this result, to generalize Brauer's result on elementary subgroups. Define $\bigotimes_{R}(G)=\{V \leqq G \mid$ there exists $N \unlhd V$ with $V / N$ elementary and $N$ a $p$-group for some $p$ with $p R \neq R\}$. Then any $R G$-module is quasi- $\xi_{R}(G)$-induced. One can also deduce intermediate statements, corresponding to the Bermann-Witt Theorem on $K$-elementary subgroups. There is still another way to generalize the above theorems: For any family $\mathfrak{U}$ of subgroups of $G$ 
define an $R G$-module $M$ to be $\mathfrak{U}$-projective, if $M$ is a direct summand in $\bigoplus_{U \in \mathfrak{u}}\left(\left.M\right|_{U}\right)^{U \rightarrow G}$. One can develop a theory of $\mathfrak{U}$-projective $R G$ modules completely analogous to D. G. Higman's theory in the case $\mathfrak{U}=\{U\}$ and one can also define for any $R G$-module $M$ its family of vertices-corresponding to Green's theory, i.e. for any $R G$-module $M$ there exists a family of subgroups $\mathfrak{U}(M)$ such that $M$ is $\mathfrak{B}$-projective if and only if $\overline{\mathfrak{B}} \geqq \mathfrak{U}(M)$ (昜 as before the subconjugate closure of $\mathfrak{W})$ and all subgroups in $\mathfrak{u}(M)$ are $p$-groups for various primes $p$ with $p R \neq R$. And one can prove that two $\mathfrak{U}$-projective $R G$-modules $M$ and $N$ are weakly isomorphic, if their restrictions $M \mid V$ and $N \mid V$ are weakly isomorphic for all $V \leqq G$ which contain a normal $p$-subgroup $N \unlhd V$ with $N \in \overline{\mathfrak{u}}, V / N$ cyclic and $p R \neq R$. In fact one proves Theorem 1 by using the above statement in some kind of complete induction argument, starting with $\mathfrak{U}=\{E\}$, the trivial subgroup. There are corresponding generalizations of the other statements.

Free University of Berlin and

Institute for Advanced Study, Princeton, New Jersey 08540 